\title{
Small molecule therapeutics for COVID-19: Repurposing of inhaled furosemide
}

\author{
Zhiyu Wang ${ }^{1,2}$, Yanfei Wang ${ }^{1}$, Prachi Vilekar ${ }^{1}$, Seung-Pil Yang ${ }^{1}$, Mayuri Gupta $^{1}$, Myong In Oh ${ }^{1}$, Autumn Meek $^{1}$, \\ Lisa Doyle ${ }^{1}$, Laura Villar ${ }^{1}$, Anja Brennecke ${ }^{1}$, Imindu Liyanage ${ }^{1,3}$, Mark Reed ${ }^{1,4}$, Christopher Barden ${ }^{1}$, Donald F \\ Weaver Corresp. 1, 2, 3 \\ ${ }^{1}$ Krembil Research Institute, University Health Network, Toronto, Ontario, Canada \\ 2 Faculty of Pharmacy, University of Toronto, Toronto, Ontario, Canada \\ 3 Faculty of Medicine, University of Toronto, Toronto, Ontario, Canada \\ 4 Department of Pharmacology and Toxicology, University of Toronto, Toronto, Ontario, Canada \\ Corresponding Author: Donald F Weaver \\ Email address: dweaver@uhnres.utoronto.ca
}

The novel coronavirus SARS-CoV-2 has become a global health concern. The morbidity and mortality of the potentially lethal infection caused by this virus arise from the initial viral infection and the subsequent host inflammatory response. The latter may lead to excessive release of pro-inflammatory cytokines, IL- 6 and IL-8, as well as TNF- $\alpha$ ultimately culminating in hypercytokinemia ("cytokine storm"). To address this immuno-inflammatory pathogenesis, multiple clinical trials have been proposed to evaluate anti-inflammatory biologic therapies targeting specific cytokines. However, despite the obvious clinical utility of such biologics, their specific applicability to COVID-19 has multiple drawbacks, including they target only one of the multiple cytokines involved in COVID-19's immunopathy. Therefore, we set out to identify a small molecule with broad-spectrum anti-inflammatory mechanism of action targeting multiple cytokines of innate immunity. In this study, a library of small molecules endogenous to the human body was assembled, subjected to in silico molecular docking simulations and a focused in vitro screen to identify anti-proinflammatory activity via interleukin inhibition. This has enabled us to identify the loop diuretic furosemide as a candidate molecule. To pre-clinically evaluate furosemide as a putative COVID-19 therapeutic, we studied its anti-inflammatory activity on RAW264.7, THP-1 and SIM-A9 cell lines stimulated by lipopolysaccharide (LPS). Upon treatment with furosemide, LPS-induced production of pro-inflammatory cytokines was reduced, indicating that furosemide suppresses the M1 polarization, including IL- 6 and TNF- $\alpha$ release. In addition, we found that furosemide promotes the production of anti-inflammatory cytokine products (IL-1RA, arginase), indicating M2 polarization. Accordingly, we conclude that furosemide is a reasonably potent inhibitor of IL- 6 and TNF- $\alpha$ that is also safe, inexpensive and well-studied. Our pre-clinical data suggest that it may be a candidate for repurposing 
as an inhaled therapy against COVID-19. 
1 Small molecule therapeutics for COVID-19: Repurposing of inhaled furosemide

3

4

5

6

7

8

9

12

Zhiyu Wang ${ }^{1,2}$, Yanfei Wang ${ }^{1}$, Prachi Vilekar ${ }^{1}$, Seung-Pil Yang ${ }^{1}$, Mayuri Gupta ${ }^{1}$, Myong $\mathrm{In} \mathrm{Oh}^{1}$, Autumn Meek ${ }^{1}$, Lisa Doyle ${ }^{1}$, Laura Villar ${ }^{1}$, Anja Brennecke ${ }^{1}$, Imindu Liyanage ${ }^{1,3}$, Mark Reed ${ }^{1,4}$, Christopher Barden ${ }^{1}$, Donald F. Weaver ${ }^{1,2,3}$

${ }^{1}$ Krembil Research Institute, University Health Network, Toronto, Canada

${ }^{2}$ Faculty of Pharmacy, University of Toronto, Ontario, Canada

${ }^{3}$ Faculty of Medicine, University of Toronto, Ontario, Canada

${ }^{4}$ Department of Pharmacology and Toxicology, University of Toronto, Ontario, Canada

Corresponding author: Donald F. Weaver ${ }^{1}$

Email address: dweaver@uhnres.utoronto.ca 
16

17

18

19

20

21

22

23

24

25

26

27

28

29

30

31

32

33

34

35

36

37

38

39

40

\section{Abstract}

The novel coronavirus SARS-CoV-2 has become a global health concern. The morbidity and mortality of the potentially lethal infection caused by this virus arise from the initial viral infection and the subsequent host inflammatory response. The latter may lead to excessive release of pro-inflammatory cytokines, IL-6 and IL-8, as well as TNF- $\alpha$ ultimately culminating in hypercytokinemia ("cytokine storm"). To address this immuno-inflammatory pathogenesis, multiple clinical trials have been proposed to evaluate anti-inflammatory biologic therapies targeting specific cytokines. However, despite the obvious clinical utility of such biologics, their specific applicability to COVID-19 has multiple drawbacks, including they target only one of the multiple cytokines involved in COVID-19's immunopathy. Therefore, we set out to identify a small molecule with broad-spectrum anti-inflammatory mechanism of action targeting multiple cytokines of innate immunity. In this study, a library of small molecules endogenous to the human body was assembled, subjected to in silico molecular docking simulations and a focused in vitro screen to identify anti-pro-inflammatory activity via interleukin inhibition. This has enabled us to identify the loop diuretic furosemide as a candidate molecule. To pre-clinically evaluate furosemide as a putative COVID-19 therapeutic, we studied its anti-inflammatory activity on RAW264.7, THP-1 and SIM-A9 cell lines stimulated by lipopolysaccharide (LPS). Upon treatment with furosemide, LPS-induced production of pro-inflammatory cytokines was reduced, indicating that furosemide suppresses the M1 polarization, including IL-6 and TNF- $\alpha$ release. In addition, we found that furosemide promotes the production of anti-inflammatory cytokine products (IL-1RA, arginase), indicating M2 polarization. Accordingly, we conclude that furosemide is a reasonably potent inhibitor of IL-6 and TNF- $\alpha$ that is also safe, inexpensive and well-studied. Our pre-clinical data suggest that it may be a candidate for repurposing as an inhaled therapy against COVID-19. 


\section{Introduction}

42 COVID-19, a potentially lethal infection caused by the SARS-CoV-2 virus, has emerged as a 43 public health crisis of global concern. Currently, there are no effective curative treatments for

44 COVID-19, affording little patient recourse beyond supportive care (Cortegiani et al. 2020). The morbidity and mortality of COVID-19 arise from two competing pathological processes, the initial viral infection and the subsequent host inflammatory response, the latter of which may

47 lead to excessive release of pro-inflammatory cytokines (interleukins [e.g. IL-6, IL-8] or noninterleukins $[$ e.g. TNF- $\alpha]$ ), and may culminate in hypercytokinemia (“cytokine storm”), a selftargeting inflammatory response syndrome (Conti et al. 2020; Mehta et al. 2020; Qin et al. 2020; Huang et al. 2020). Reflecting these dichotomous disease processes, current therapeutic

51 development strategies may be categorized into two broad groups: anti-viral and anti-

52 inflammatory. Arguably, truly effective treatments may require both agents, since an antiviral will fail to suppress uncontrolled pro-inflammatory cytokine release once the process has been 54 triggered. To address the COVID-19 immuno-inflammatory pathogenesis, multiple clinical trials have been proposed to evaluate anti-inflammatory biologic therapies targeting specific cytokines. Agents under consideration include tocilizumab (Peking University First Hospital 2020; Tongji Hospital et al. 2020; National Cancer Institute Naples 2020) and sarilumab (Regeneron

59 Pharmaceuticals and Sanofi 2020), both monoclonal antibodies that target the IL-6 pathway 60 (Sebba 2008; Boyce et al. 2018), as well as adalimumab, which binds with specificity to TNF- $\alpha$ 61 (Furst et al. 2003). Data from a Chinese study in which tocilizumab was given to 21 patients with severe COVID-19 reported that the patients "improved remarkably" with 13 patients being

63 discharged within 14 days or less following treatment, six patients within $14-21$ days and only 
64 two patients stayed in the hospital for more than 21 days (Xu et al. 2020). However, despite the

65 obvious clinical utility of such biologics for many disorders, their specific applicability to

66 COVID-19 is hampered by various issues: they target only one of the multiple cytokines

67 implicated in COVID-19's immunopathy; if administered systemically, they can predispose

68 patients to secondary infections or other toxicities, such as hepatoxicity; and they may be

69 expensive to mass produce and distribute. Therefore, they are of limited utility in the context of a 70 global pandemic.

Accordingly, we set out to identify a small molecule with the following properties: broadspectrum anti-inflammatory mechanism of action targeting cytokines of innate immunity; low toxicity and excellent safety profile; chemically stable; easily stored and administered; able to be rapidly adopted in clinical settings worldwide; and, widespread availability with inexpensive and efficient means of production. As described herein, a systemic series of in silico and in vitro studies have enabled us to identify furosemide as a candidate molecule.

77

\section{Materials \& Methods}

\section{In Silico Studies}

Molecular docking simulations: Molecular docking simulations on the endogenous molecule (1136, created in-house through literature search) and known drug datasets (1768, taken from drugbank.ca) against Il-6 and TNF- $\alpha$ were carried out in two steps. The first step involved placement of the endogenous or ligand molecule in an identified binding pocket

84 (docking) of IL-6 and TNF- $\alpha$. In the second step, the calculation of the binding energies of the 85 docked molecules (scoring) was completed. 
Preparation of TNF- $\alpha$ and IL-6 $3 D$ protein structures: The TNF- $\alpha$ structure (PDB code:

87

2AZ5) and IL-6 structure (PDB code: 1ALU) were selected for molecular docking simulations in this work. We have employed these X-ray crystallographic structures because of three underlying motivations: 1 . These X-ray crystallographic structures are solved for human IL-6 and TNF- $\alpha$ proteins. 2. Both of these X-ray structures have been solved at high resolution i.e. 1ALU for IL-6 at $1.90 \AA$ and $2 \mathrm{AZ} 5$ for TNF- $\alpha$ at $2.10 \AA$, which minimizes the error while solving the structure from electron density maps. 3. The ligand molecules did not involve any metal atoms, which can otherwise bias the active site binding residues.

The downloaded PDB structures were loaded into Molecular Operating Environment (MOE 2019.0101) software (MOE, 2019). The loaded crystal structure was prepared employing the 'QuickPrep Panel' in MOE, which contains the 'structure preparation' feature. The 'Protonate3D' function was used to optimize ionization states of the added hydrogen atoms. Water molecules, which were present $4.5 \AA$ away from ligand or receptor, were deleted. The MFF94x force field, with RMS gradient of $0.05 \mathrm{kcal} / \AA$, was used for energy minimization (Panigrahi \& Desiraju 2007). A Ramachandran plot was used to confirm the geometric and stereochemical qualities of the TNF- $\alpha$ and IL- 6 protein structures.

Endogenous molecules and known drugs dataset curation: The datasets of endogenous molecules (1136) and drugs (1768) were prepared using MOE software. Two functions 'wash at dominant $\mathrm{pH}$ of 7.4' and 'energy minimize' were used to obtain an energy minimized molecular form of molecules present at physiological $\mathrm{pH}$ of 7.4.

Binding site selection and docking simulations: The active site of IL-6 and TNF-alpha was identified using the 'MOE-site Finder' module. The 'Triangle Matcher' placement method and 'London $\mathrm{dG}$ ' scoring functions were used for docking simulations. The poses from ligand 
109 conformations were generated in the Placement phase. After placement of poses, the refinement

110 was done with the 'GBVI/WSA dG' scoring method using 'induced fit' option. Induced fit

111 refinement allows both the ligand molecule and protein active site to move freely to facilitate

112 residue alignment during docking simulations. Thirty docked poses of placement retention

113 simulation and 10 poses of refinement step were retained. The docking pose corresponding to the

114 highest score for an endogenous or a drug molecule was considered for comparison of binding

115 affinity within datasets. Further details of descriptor calculation and docking simulations are

116 provided in our recent publications (Gupta et al. 2019; Gupta et al. 2020) and in supporting

117 information.

118 Docking method validation: Validation of the docking protocol was carried out by re-

119 docking the co-crystallized ligand in the active sites of the IL- 6 and TNF- $\alpha$ protein receptors.

120 The following parameters were confirmed for validating the accurate docking protocol:

121 1. Molecular superposition: The lowest energy docked conformation of a native ligand for

122 IL-6 and TNF- $\alpha$ is overlaid relative to the co-crystallized ligand present in the X-ray

123 structure of the protein; the root-mean-square deviation (RMSD) has been calculated and

124 is taken as an evaluation criterion for the validation of docking simulations. An RMSD

125 value of $<2.0 \AA$ between docked and crystallographic ligand conformation confirms

126 successful docking (Plewczynski et al. 2011; Ramírez and Caballero 2018).

127

2. Ligand interactions with the active site: The docking protocol is validated if the docked ligand interacts with the active site of the protein similar to the corresponding cocrystallized ligand conformer and exhibits the same interactions with active site amino acid residues (Plewczynski et al. 2011; Ramírez and Caballero 2018). 


\section{In Vitro Studies}

Inflammation activation on RAW264.7 macrophage: RAW264.7 cells were purchased

134 from ATCC and maintained in Dulbecco's Modified Eagle's Medium (DMEM) containing foetal 135 bovine serum (FBS) at a final concentration of 10\%. RAW264.7 cells were seeded in 12-well 136 plates at $0.25 \times 10^{6}$ cells/well seeding density, one day before experiments. To activate the cells,

137 cell culture medium was changed to a lipopolysaccharide (LPS) and Interferon $\gamma$ (IFN $\gamma$ )

138 containing medium with dimethyl sulfoxide (DMSO) or furosemide at required concentrations, 139 followed by $24 \mathrm{~h}$ incubation at $37^{\circ} \mathrm{C}$; conditioned media and lysates were harvested for analysis.

140 Inflammation activation on THP-1 monocytic cells: THP-1 cells (ATCC) were

141 maintained in RPMI 1640 medium supplemented with 2-mercaptoethanol at a final concentration 142 of $0.05 \mathrm{mM}$ and FBS at a final concentration of $10 \%$. THP-1 cells were seeded in each well of a 143 12-well tissue culture plate at a density $0.5 \times 10^{6}$ cells $/ \mathrm{mL}$, one day before experiments. THP-1

144 monocytes were differentiated by $150 \mathrm{nM}$ PMA (phorbol 12-myristate 13-acetate) for $24 \mathrm{~h}$. Cells 145 were then treated with LPS+IFN $\gamma$ with DMSO or furosemide at the required concentration, 146 followed by $48 \mathrm{~h}$ incubation; conditioned media and lysates were collected for analysis.

147 Nitric oxide (NO) production by Griess assay: Nitric oxide production from the 148 conditioned media of RAW264.7 and SIM-A9 cell cultures was examined by a Griess assay.

149 Conditioned medium and sulfanilamide were mixed in a microwell plate to form a transient 150 diazonium salt. Then, $N$-naphtyl-ethylenediamine was added to all wells to form a stable azo 151 compound by incubating for 5-10 min in a dark at room temperature. The absorbance was 152 measured between $520 \mathrm{~nm}$ and $550 \mathrm{~nm}$. The concentration of NO production was quantified by 153 being plotted against a standard curve. 
ELISA kits following the manufacturer's instructions. Briefly, the high-binding plates were

156

157

158

159

160

161

162

163

164

165

166

167

168

169

170

171

172

173

174

175

176

coated at $100 \mu \mathrm{L} /$ well with diluted capture antibodies $(1: 250)$ at $4{ }^{\circ} \mathrm{C}$ overnight. The coated plates were then blocked with the diluent for $1 \mathrm{~h}$ before assay. Each sample was diluted accordingly and added to the plates for a $2 \mathrm{~h}$ incubation period at room temperature. Plates were then washed with $250 \mu \mathrm{L} /$ well PBS with $0.05 \%$ Tween-20 and incubated with detection antibodies (1:250 in assay diluent) for $1 \mathrm{~h}$ at room temperature. After another washing step, 1:250 diluted avidin-HRP (horseradish peroxidase) was added and incubated for $30 \mathrm{~min}$. Next, $100 \mu \mathrm{L}$ TMB-substrate (3,3',5,5'-tetramethylbenzidine) was added and the plate was incubated in dark until the signal was sufficiently developed. The final concentration of TMB substrate solution is $200 \mu \mathrm{g} / \mathrm{mL}$. The reaction was stopped with $50 \mu \mathrm{L}$ of $2 \mathrm{~N}$ sulfuric acid. Absorbance was measured at $450 \mathrm{~nm}$ with a correction of $570 \mathrm{~nm}$ using a plate reader.

Western blotting: Cells were washed twice with ice-cold PBS and harvested in RIPA buffer supplemented with a protease inhibitor cocktail. The whole-cell extracts were then centrifuged at $22,000 \mathrm{xg}$ for $20 \mathrm{~min}$ at $4{ }^{\circ} \mathrm{C}$ to remove cell debris. Protein concentrations were quantified using a Micro BCA protein assay kit. The absorbance was measured at $595 \mathrm{~nm}$ using a microplate reader. Equal amounts of cellular protein were separated by sodium dodecyl sulfatepolyacrylamide gel electrophoresis (SDS-PAGE) and transferred onto polyvinylidene difluoride (PVDF) membranes at $100 \mathrm{~V}$ for $90 \mathrm{~min}$. The SDS-PAGE was performed with $10 \%$

polyacrylamide gel for iNOS and $12.5 \%$ for pro-IL-1 $\beta$. The membranes were blocked for $1 \mathrm{~h}$ in Tris-buffered saline (TBS), pH 7.4, with 0.1\% Tween-20 (TBS-T) containing 10\% skim milk. The membrane blot was then incubated overnight at $4{ }^{\circ} \mathrm{C}$ with primary antibodies against iNOS (1:1000), IL-1 $\beta$ (1:1000), actin (1:5000) and GAPDH (1:5000) in TBS-T containing 5\% skim 
177 milk. The membrane was washed with TBS-T 3 x 10 mins and incubated with goat anti-rabbit

178 IgG-horseradish peroxidase (1:5,000) for 1 hour. After the washing step, the immunoblotting 179 was visualized by chemiluminescence HRP-substrate.

180 Flow cytometry: Cells were harvested and re-suspended with staining buffer. Cells were 181 stained with antibody $(1: 100)$ by incubating at $4{ }^{\circ} \mathrm{C}$ for $30 \mathrm{~min}$ in the dark. Stained cells were 182 centrifuged, and the supernatant was discarded. The cell pellets were then re-suspended in cell 183 flow buffer, transferred to FACS tubes and analysed by flow cytometry within $48 \mathrm{~h}$. To all cells 184 in the experiment, Fc blocker was added.

Inflammation activation on SIM-A9 cells: SIM-A9 (ATCC) cells were maintained in Dulbecco's modified eagle medium: nutrient mixture F-12 (DMEM-F12) with 10\% foetal bovine serum, 5\% horse-serum and antibiotic-antimycotic. SIM-A9 cells were seeded $24 \mathrm{~h}$ before the experiment. Culturing medium was replaced with DMEM-F12 medium containing 5\% FBS + $2.5 \%$ horse serum with required LPS concentration (final volume is $1 \mathrm{~mL} /$ well). The conditioned media and lysates were harvested for cytokine and cell marker examination.

Statistical analysis: Data are presented as mean \pm SD or \pm SEM. Statistical analysis was performed with GraphPad Prism software version 6.01c, applying a two-tailed unpaired t-test. A $p$-value of $>0.05$ was considered significant.

\section{Results}

\section{Identifying an Initial Hit}

A screening strategy was devised for identifying an initial compound with potential broad- 
200 of either up- or down-regulation of innate immune processes. Accordingly, we sought to identify 201 an endogenous compound as an initial molecular platform around which to devise a therapeutic.

202 A library of 1,136 small molecules endogenous to the human body was assembled, subjected to 203 in silico molecular docking simulations and a focused in vitro screen to identify anti-pro204 inflammatory activity via interleukin inhibition.

205 Multiple compounds within the tryptophan metabolic pathway were identified, both 206 indoleamine and anthranilate metabolites. In particular, 3-hydroxyanthranilic acid (3-HAA) was 207 found to demonstrate significant anti-inflammatory potential (in accord with previous studies by 208 others, such as Lee et al. (2013), who have shown significant activity of 3-HAA in inhibiting IL2096 and TNF- $\alpha$ ). Regrettably, 3-HAA is a small polar molecule with poor drug-like properties and 210 is not an approved therapeutic for human use.

211

\section{Converting Hit to Drug-Like Anthranilate Compound}

213 The task of converting 3-HAA to a drug or drug-like compound can be addressed via two 214 approaches: 1) synthesis of new chemical entities with drug-like properties based on the 3-HAA 215 scaffold, or 2) identification of known drugs with structural properties similar to the 3-HAA 216 scaffold with the goal of repurposing. Because of the urgency imposed by the unfolding 217 pandemic, approach 2 was selected to provide a short-term solution. Accordingly, 1,768 known drugs were computationally evaluated for anthranilate 219 structural components. This screen identified mefenamic acid ( $N$-(2,3-xylyl)-anthranilate) and 220 furosemide (4-chloro-5-sulfamoyl- $N$-furfuryl-anthranilate) as the two strongest leads. Mefenamic 221 acid is known to provide significant protection against elevated levels of TNF- $\alpha$ and IL-1 $\beta$ in 222 radiation-induced genotoxicity of human lymphocytes (Armagan et al. 2012; Hosseinimehr et al. 
223 2015); furosemide is known to significantly reduce production of IL-6, IL-8, and TNF- $\alpha$ in

224 bronchial inflammation of asthma (Prandota 2002; Yuengsrigul et al. 1999). This screen also

225 identified other loop diuretics structurally related to 3-HAA and furosemide, including

226 bumetanide, piretanide and azosemide (Fig. 1).

227

Arising from the observation that furosemide can reduce bronchial inflammation, can be

228

229

230

231

232

233

234

235

236

237

238

239

240

241

242

243 244 production by furosemide, we determined the expression level of inducible nitric oxide synthase 245 (iNOS) which produces NO in response to inflammatory stimulations. We stimulated RAW264.7

\section{In Vitro Assessment of Lead Anthranilate Compound}

To pre-clinically evaluate furosemide as a putative COVID-19 therapeutic, a series of in vitro efficacy assessments was performed to investigate its anti-inflammatory properties. First, we investigated if furosemide could reduce the release of pro-inflammatory cytokines induced by LPS in macrophage cell line. RAW264.7 macrophages were stimulated with LPS in the presence or absence of furosemide and the level of TNF- $\alpha$ and NO were measured from the conditioned media using ELISA and Griess assay, respectively. The results in Fig. 2 (A) and (B) show that LPS induces the production of NO and TNF- $\alpha$, indicating macrophage polarization to an M1 proinflammatory phenotype.

When cells were treated with LPS in the presence of $25 \mu \mathrm{M}$ of furosemide, the production of NO and TNF- $\alpha$ significantly decreased. To further investigate the reduction of NO 
246 macrophages with LPS and IFN $\gamma$. The expression of the iNOS was significantly induced by

247 stimulation with LPS and IFN $\gamma$, as shown by the Western blot results in Fig. 2 (C). We found

248 that furosemide was able to suppress the expression of iNOS during LPS and IFN $\gamma$ induced

249 stimulation. Densitometry analysis showed that normalized iNOS/GAPDH ratio was reduced

250 from 1 to 0.88 by furosemide.

251 LPS is recognized by the cell surface pattern-recognition receptors such as the toll-like

252 receptor 4 protein (TLR4) and triggers downstream signalling pathways. LPS induces expression

253 of the pro-inflammatory cytokine IFN $\gamma$. IFN $\gamma$ is known to increase TLR4 expression which may

254 then promote the response to LPS stimulation. We investigated the effect of furosemide on LPS-

255 induced TLR4 expression in RAW264.7 macrophage cells using flow cytometry. As shown in

256 Fig. 3, LPS increased the TLR4+ cell population significantly.

257

Whereas IL-4, an anti-inflammatory cytokine, barely induced TLR expression from the

258

259

260

261

262

263

264

265

266

267

268

cells. Interestingly, furosemide completely blocked LPS-induced TLR4 expression, suggesting the possible involvement of furosemide in the LPS- or IFN $\gamma$-induced inflammation.

As the next stage of macrophage activation, we studied the activity of furosemide on the expression of IL-1 $\beta$ which plays a key role in modulating inflammatory response, as a downstream pro-inflammatory marker of the TLR4 signalling pathway by using differentiated THP-1 monocytes. We analysed the expression of IL-1 $\beta$ precursor protein (pro-IL-1 $\beta$ ) from THP-1 macrophage cells by using Western blot analysis. Furosemide, as shown in Fig. 4, significantly decreases the expression of pro-IL-1 $\beta$ in differentiated THP-1 cells, demonstrating yet another inflammatory cytokine targeted by furosemide. To further explore the effect of furosemide on different macrophages, we next tested it on SIM-A9 cells. We stimulated SIM-A9 macrophages with LPS and analysed the release of pro-inflammatory markers such as NO, IL-6 
269 and TNF- $\alpha$. The results in Fig. 4 show that LPS induced the production of NO, IL-6 and TNF- $\alpha$

270 from SIM-A9 cells. Similar to RAW264.7 cell results, furosemide significantly reduced the

271 production of all these pro-inflammatory markers from SIM-A9 cells as well.

272 We have tested three different macrophage cell lines for the effects of furosemide on pro-

273 inflammatory markers. Furosemide consistently reduced pro-inflammatory markers such as NO

274 production, secretion of IL-6, TNF- $\alpha$ and expression of pro-IL-1 $\beta$ from different macrophage

275 cell lines, implying that furosemide has broad inhibitory activity against pro-inflammatory 276 cytokines.

277

Then, we evaluated if furosemide exhibits any effects on anti-inflammatory cytokines.

278 We measured the levels of anti-inflammatory cytokines from the conditioned medium of

279 differentiated THP- 1 cells after $48 \mathrm{~h}$ of stimulation with LPS and IFN $\gamma$ by Multiplex assay using

280 flow cytometry. Furosemide induces the expression of IL-4, interleukin-1 receptor antagonist

281 (IL-1RA) and arginase, which are anti-inflammatory markers, suggesting the polarization of

282 THP-1 macrophage to an M2 phenotype (Fig. 5).

283 Together with the experiments discussed above, these results show that furosemide

284 inhibits the expression of M1 pro-inflammatory markers and promotes the expression of M2

285 anti-inflammatory markers. Thus, furosemide is a broad-spectrum anti-inflammatory drug

286 candidate targeting multiple cytokines.

287

\section{In Silico Mechanism of Action Simulations}

289 The molecular mechanism of action whereby 3-HAA and furosemide inhibit IL-6 and TNF- $\alpha$

290 activity is unknown at this time and lies outside the scope of the present study. Interestingly, in

291 an in silico screen of possible binding sites for 3-HAA and related drug anthranilates, we noted 
292 favourable interactions with both the IL-6 and TNF- $\alpha$ proteins. Whilst it is improbable that such 293 interactions are completely responsible for the observed anti-inflammatory effects, this notable 294 observation is worthy of future experimental evaluation.

295 The docking protocol needs to be validated before any conclusive in silico results can be 296 obtained. To validate the docking methodology, co-crystalized ligands for TNF- $\alpha$ and IL-6 have 297 been re-docked into the active sites of the corresponding proteins. Table 1 shows the re-docking 298 parameters that have been evaluated for confirmation of accuracy of the employed docking 299 protocol. IL-6 (PDB code: 1ALU) has been re-docked with tartaric acid.

300 After re-docking simulations, the docked conformer of tartaric acid is overlaid with the co301 crystallized ligand in the PDB structure. Figure 6 (A) represents the overlay of the docked pose 302 and the co-crystallized ligand conformer. The RMSD between the docked conformer and co303 crystallized ligand is $0.6114 \AA$, which is $<2.5 \AA$ as recommended for successful docking method 304 confirmation. Furthermore, all the amino acid residues which are interacting with the native IL-6 305 binding pocket (Arg179, $\operatorname{Arg} 182$ and Gln175) are shown to be interacting with the docked 306 conformer of the tartaric acid in the re-docking simulation (as shown in Fig. 6 (B)).

307 This further confirms that the re-docking method is able to accurately predict the binding mode 308 and the active site of IL-6. The TNF- $\alpha$ dimer inhibitor has also been re-docked with TNF- $\alpha$ for 309 validation of the docking method. The overlay of the docked ligand with the co-crystallized 310 ligand conformation is presented in Fig. 7 (A). From Fig. 7 (A) it can be seen that the TNF- $\alpha$ 311 dimer inhibitor 307 is superposed with the co-crystallized ligand conformer with an RMSD value 312 of $0.1677 \AA$, which validates the docking simulation methodology for TNF- $\alpha$. The ligand 313 interaction diagram of the docked conformer of TNF- $\alpha$, is shown in Fig. 7 (B). The amino acid 314 residues present within $4 \AA$ of the active site are labelled i.e. Leu57, Tyr59, Ser60, Leu120, 
315 Gly121, Gly122, Tyr151. These residues are present in the active site of TNF- $\alpha$ dimer in the 316 vicinity of co-crystallized ligand. The re-docking simulations of co-crystallized ligands for IL-6 317 and TNF- $\alpha$ confirm the accuracy of the docking methodology employed in this study.

318 The computational simulations of 3-HAA and furosemide with TNF- $\alpha$ and IL-6 are briefly 319 outlined below. The binding of 3-HAA in IL-6 and TNF- $\alpha$ active sites is presented in Fig. 8. The 320 docking score (S) of minimum energy pose of 3-HAA in IL-6 is -4.620 , and in TNF- $\alpha$ is -4.487 . 321 In Figs. 8 (A) and (C), the ligand 3-HAA is shown in yellow and amino acid residues of binding 322 pocket have been labelled. Figures 8 (B) and (D) represent the ligand interaction diagrams of 3323 HAA with active site residues of TNF- $\alpha$ and IL-6. 3-HAA interacts with the Arg179 and Arg182 324 in IL-6 and docks in the TNF- $\alpha$ dimer site with residues Tyr119, Tyr59, Ser60, Leu120, Gly121 325 and Tyr 151 . This confirms that 3-HAA interacts with the IL-6 and TNF- $\alpha$ in the same binding 326 site as observed in re-docking simulations of co-crystallized ligands for the corresponding 327 proteins.

Figure 9 presents docking simulations of furosemide with TNF- $\alpha$ and IL-6, respectively. In Figs. 9 (A) and (C), furosemide is shown in yellow and the amino acid residues of the binding 330 site are labelled. The TNF- $\alpha$ protein complex (PDB: 2AZ5) contains four identical chains having 331148 amino acids each and two bound ligands. The A and B chains were retained (coloured 332 orange and purple) and the co-crystalized ligand was removed while preparing the protein 333 structure for docking.

Figure 9 (A) shows the minimum energy docking pose of furosemide in the TNF- $\alpha$ active 335 site. The amino acid residues Leu B57, Tyr B59, Gly B121, Tyr A151, Tyr A119 and Leu A120 are involved in forming hydrogen bonding and $\pi-\pi$ interactions (arene-arene, arene- $\mathrm{H}$, arenecation) with furosemide in minimum energy docked poses. The docking score of the minimum 
338 energy pose is -6.0854 . The similar mode of binding in the active site of TNF- $\alpha$ as of co-

339 crystallized ligand (as shown in Fig. 7 (D)) and favourable binding energy indicates that

340 furosemide fits well into the active site of TNF- $\alpha$ and inhibits its activity.

341 Figure 9 (C) and (D) show the minimum energy docking pose of furosemide in the

342 protein structure of human IL-6 (PDB: 1ALU), having 186 amino acid residues and a co-

343 crystallized ligand (tartaric acid). The co-crystallized ligand had been removed during the

344 structure preparation step of the IL-6 protein. Furosemide interacts with Arg 182, Gln 175, Leu

34533 and Arg 179 in most of the minimum energy poses, which agrees well with the IL-6 co-

346 crystallized ligand tartaric acid binding mode. The docking score (S) of the minimum energy

347 pose of furosemide with IL-6 is -5.1343 , which computationally supports the ability of

348 furosemide to inhibit IL-6 activity.

349 Discussion

350 The pathogenic mechanisms of COVID-19 morbidity and mortality are diverse, though immuno-

351 inflammatory contributions are likely a central player. It is now appreciated that COVID-19

352 afflicted individuals with major respiratory symptoms have pathologically elevated levels of pro-

353 inflammatory cytokines including IL-6, IL-8 and TNF- $\alpha$ (Conti et al. 2020; Mehta et al. 2020;

354 Qin et al. 2020; Huang et al. 2020). A logical therapeutic approach to the management of

355 COVID-19 thus includes a need to modulate immunotoxicity.

356 Tryptophan and its metabolites, particularly via the indoleamine-2,3-dioxygenase

357 initiated pathway, have a previously described role as endogenous modulators of innate

358 immunity. Of these various metabolites, 3-HAA has been identified to exhibit a significant anti-

359 inflammatory ability to suppress inflammation mediated via multiple pro-inflammatory

360 interleukin cytokines, including IL-1 $\beta$, IL-6, IL-8 and TNF- $\alpha$ (Lee et al. 2013). This motivated 
361 our search for an anthranilate-based 3-HAA-like agent from a library of known drugs;

362 furosemide emerged as a possible candidate from this search.

363 In this study, as part of its pre-clinical evaluation, we studied furosemide's anti-

364 inflammatory activity on multiple macrophage cell lines involved in innate immunity. As pattern

365 recognition receptors, TLRs contribute to the recognition of the molecules that are commonly

366 shared by pathogens, such as LPS and viral nucleotides (Alexopoulou et al. 2001). The

367 activation of TLRs triggers downstream signalling through the MyD88-dependent pathway and

368 eventually induces the activation of nuclear factor-kB (NF-KB) (Oeckinghaus et al. 2011). NF-

$369 \mathrm{\kappa B}$ has been shown to play an important role in coronavirus infections. For instance, NF-кB

370 activation was identified in the lungs of SARS-CoV infected mice and triggered the production

371 of pro-inflammatory cytokines, such as TNF- $\alpha$ (DeDiego et al. 2014). However, the mechanism

372 of SARS-CoV pathogenesis is debated. An in vitro study suggested the nucleocapsid protein of

373 SARS-CoV was crucial in the pathogenesis, although this correlation is cell-specific (Liao et al.

374 2005). On the other hand, SARS-CoV lacking the envelope protein (E protein) attenuated NF-кB

375 activation and associated pro-inflammatory responses (DeDiego et al. 2014). The pandemic

376 outbreak of COVID-19 is caused by the infection of SARS-CoV-2 that shares $79.5 \%$ identity to

377 SARS-CoV (Guo et al. 2020). To investigate the potential use of furosemide as a therapy for

378 COVID-19, we developed in vitro assays using LPS as exogenous stimulation to induce

379 inflammatory responses. LPS-induced TLRs activation is well studied: it interacts with TLRs

380 and triggers NF-кB activation followed by pro-inflammatory responses (Lu et al. 2008). In our in

381 vitro assays, we aimed to ascertain if LPS induces a similar 'cytokine storm' as SARS-CoV-2

382 infection and if furosemide inhibits the production of pro-inflammatory cytokines or promotes

383 the secretion of anti-inflammatory cytokine products. 

inflammatory phenotype or an M2 anti-inflammatory phenotype. We investigated if furosemide inhibits the production of pro-inflammatory cytokines (M1) or promotes the secretion of anti-

387 388 inflammatory cytokines (M2) using various macrophage cell lines including RAW264.7, THP-1 and SIM-A9. Upon stimulation, these cell lines initiated an immune response by producing cellular stress signals and secreting pro-inflammatory cytokines. These pro-inflammatory markers were reduced upon treatment with furosemide, indicating that furosemide suppresses the M1 polarization, including NO, IL-6 and TNF- $\alpha$. More importantly, our study results demonstrated furosemide promotes the production of anti-inflammatory cytokine products (IL1RA, arginase), indicating M2 polarization. All these results strongly suggest the potential application of furosemide as an immunomodulating agent for such disease conditions in which the inflammatory burden of patients increases suddenly. It also suggests that furosemide can be used for treating COVID-19 in which the sudden increase of pro-inflammatory cytokines is part of the disease pathogenesis.

Furosemide is a small molecule with a molecular weight of $330.75 \mathrm{~g} / \mathrm{mol}$ and relatively low lipophilicity $(\log \mathrm{P}=2.03)$ (Hardman et al. 2001). Although the drug has low water solubility at $\mathrm{pH} 7$, furosemide can be formulated in weakly basic buffer solution ( $\mathrm{pH} 8$ ) to achieve $10 \mathrm{mg} / \mathrm{mL}$ solutions suitable for intravenous administration. Due to the presence of a primary sulfonamide and carboxylic acid group, furosemide is highly bound to albumin with a human plasma protein binding value of $98.6 \pm 0.4 \%$. The drug has a very low volume of distribution $\left(\mathrm{V}_{\mathrm{D}}=0.13 \pm 0.06 \mathrm{~L} / \mathrm{kg}\right)$ and a relatively short half-life of $1.3 \pm 0.8 \mathrm{~h}$ (Hardman et al. 2001). These pharmacokinetic parameters along with high plasma protein binding equates to low tissue distribution with furosemide being retained with the blood. Alveolar macrophages are 
407 front line innate immune cells, playing a crucial role in the maintenance of lung homeostasis and 408 lung tissue defence through various immune responses. Tissue-resident alveolar macrophages are 409 derived from the foetal liver and populate the alveoli shortly after birth. These macrophages are 410 self-renewing and persist over the life span. However, exposure to environmental challenges and 411 injury induces recruitment of monocyte-derived alveolar macrophages from circulating 412 monocytes. The intrinsic molecular properties of furosemide are ideal for targeting macrophages 413 recruited from the blood stream prior to their tissue distribution.

414 Furosemide exhibits a large therapeutic window and is listed on the WHO's List of 415 "Essential Medicines"; it is readily available worldwide, is easily manufactured, and has a long 416 record of safety and efficacy when given orally or intravenously. More importantly, furosemide 417 may also be administered safely by inhalation. More than 20 years ago, the concept of inhaled 418 furosemide was explored as an approach to reduce dyspnoea, primarily based on the rationale 419 that oedematous airway mast cells would be reduced in size following diuresis (Prandota 2002). 420 However, further investigations established that the mechanism of action was not related to local 421 diuretic effect or engagement of the $\mathrm{Na}^{+} / \mathrm{K}^{+}$-ATP shuttle. More recent studies have reported 422 reduction in pulmonary IL-6, IL-8, and TNF- $\alpha$ levels upon administering inhaled furosemide to 423 patients with conditions including tachypnoea (Armed Forces Hospital Pakistan 2016; University 424 of Cologne 2012), bronchopulmonary dysplasia (University of North Carolina et al. 2015), and 425 chronic lung disease (Center BIDM and Research NIoN 2014; McGill University 2016; Oxford 426 Brookes University 2015). A 2018 double-blind, placebo-controlled trial by Grogono et al. 427 (2018) evaluated inhaled nebulized furosemide (40 mg furosemide in $4 \mathrm{~mL} 0.9 \%$ saline) in 428 healthy adults, demonstrating effective relief of experimentally induced air hunger during 429 dyspnoea after multiple dosing per day with no untoward effects. Therefore, accumulated data 
430 indicate that furosemide is a cytokine-targeting anti-inflammatory, which may be safely

431 administered by inhalation multiple times per day.

432 Our in silico screening identified other loop diuretics with structural similarities to

433 furosemide. Bumetanide exhibits anti-inflammatory properties in LPS stimulated RAW264.7

434 cells, reduces LPS-induced production of cytokines following direct pulmonary administration,

435 and lowers levels of TNF- $\alpha$ production in lung-injured mice (Hung et al. 2018). Bumetanide

436 however failed to inhibit sodium metabisulfite induced bronchoconstriction in asthmatic subjects

437 (O'Connor et al. 1991). Piretanide and azosemide have also been variously studied in models of

438 cytokine-mediated inflammation and bronchoconstriction (Yeo et al. 1992). Since none of these

439 agents are in widespread clinical use, we have elected to pursue the development of furosemide

440 in COVID-19 because of its worldwide availability in the time of a global pandemic.

441 The potential use of furosemide in the anti-inflammatory treatment of COVID-19 has

442 strengths and weaknesses. Furosemide is inexpensive and available in every country in the

443 world; it is safe and has profound anti-inflammatory cytokine activity, particularly against IL-6

444 and TNF- $\alpha$. If administered orally, furosemide can produce a profound diuresis which would be

445 a clinical detriment in a febrile and potentially dehydrated person. When administered orally, the

446 pharmacokinetics of furosemide indicate that it would have primarily intra-vascular distribution,

447 suggesting greater utility early in the course of the disease, but less so in later-stage ventilator-

448 supported individuals in whom macrophage migration from bloodstream to pulmonary tissue has

449 occurred. As the disease progresses, direct administration of furosemide to the lungs by

450 nebulized delivery adequately addresses the need to have furosemide reach intra-alveolar

451 macrophages. Arguably, truly effective treatments may require both anti-viral and anti-

452 inflammatory agents since the early stages of the infection are dominated by high viral 
453 replication. However, application of an anti-inflammatory agent only, especially in the current

454 situation where an effective anti-viral is still missing, may have the potential to reduce the

455 number of patients requiring mechanical ventilation and reduce cough as a symptom which could

456 help limiting the spread of the virus.

457 Care must be taken to prevent viral contamination and bystander exposure during the aerosolized

458 administration of the drug, but this can be achieved with appropriate cautions in place. We are

459 currently pursuing a clinical study of inhaled furosemide in people with COVID-19.

460

461 Conclusions

462 COVID-19 is a pandemic threatening global health. The need to identify innovative therapeutics 463 which may be deployed rapidly and efficiently is a pharmacological priority. Furosemide is a 464 safe, inexpensive, well-studied small molecule which is a reasonably potent inhibitor of IL-6 and 465 TNF- $\alpha$ and may be administered locally to the lungs; pre-clinical data from in silico and in vitro 466 experiments suggest that it may be a candidate for repurposing as an inhaled therapy against the 467 immunopathologies of COVID-19.

468

469 Supporting Information: A list of physiochemical descriptors used as initial screening test of

470 the dataset; a list of promising drug candidates along with corresponding physiochemical

471 descriptors; Docking Score ' $S$ ' of promising drug candidates with IL-6 and TNF- $\alpha$; Figures of

472 Mefenamic acid, Bumetanide, Piretanide and Azosemide drugs docking into binding site of

473 TNF- $\alpha$ and IL-6 and Ligand interaction diagram of Mefenamic acid Mefenamic acid,

474 Bumetanide, Piretanide and Azosemide drugs in active site of TNF- $\alpha$ and IL-6. This information 475 is available free of charge on PeerJ. 


\section{References}

478 Alexopoulou L, Holt AC, Medzhitov R, Flavell RA. Recognition of double-stranded RNA and 479 activation of NF-kB by Toll-like receptor 3. Nature. 2001;413(6857):732-38.

480 doi:10.1038/35099560.

481

482

483

484

485

486

487

488

489

490

491

492

493

494

495

496

497

498

499

500

501

502

503

504

505

506

507

508

509

510

511

512

513

514

515

516
Armagan G, Turunc E, Kanit L, Yalcin A. Neuroprotection by mefenamic acid against D-serine: involvement of oxidative stress, inflammation and apoptosis. Free Radic Res. 2012;46(6):72639. doi:10.3109/10715762.2012.669836. doi:10.3109/10715762.2012.669836.

Armed Forces Hospital Pakistan. Role of Salbutamol and Furosemide in TTN. https://ClinicalTrials.gov/show/NCT03208894 2016.

Boyce EG, Rogan EL, Vyas D, Prasad N, Mai Y. Sarilumab: Review of a Second IL-6 Receptor Antagonist Indicated for the Treatment of Rheumatoid Arthritis. Ann Pharmacother. 2018;52(8):780-91. doi:10.1177/1060028018761599.

Center BIDM, Research NIoN. Aerosol Inhalation Treatment for Dyspnea - Patients. https://ClinicalTrials.gov/show/NCT02524054 2014.

Conti P, Ronconi G, Caraffa A, Gallenga C, Ross R, Frydas I, Kritas S. Induction of pro-

inflammatory cytokines (IL-1 and IL-6) and lung inflammation by Coronavirus-19 (COVI-19 or SARS-CoV-2): anti-inflammatory strategies. J Biol Regul Homeost Agents 2020;34(2). doi:10.23812/CONTI-E.

Cortegiani A, Ingoglia G, Ippolito M, Giarratano A, Einav S. A systematic review on the efficacy and safety of chloroquine for the treatment of COVID-19. J Crit Care. 2020;57:279-283. doi:10.1016/j.jcrc.2020.03.005.

DeDiego ML, Nieto-Torres JL, Regla-Nava JA, Jimenez-Guardeño JM, Fernandez-Delgado R, Fett C, Castaño-Rodriguez C, Perlman S, Enjuanes L. Inhibition of NF- $\kappa \mathrm{B}$-mediated inflammation in severe acute respiratory syndrome coronavirus-infected mice increases survival. J Virol. 2014;88(2):913-24. doi:10.1128/JVI.02576-13.

Furst DE, Schiff MH, Fleischmann RM, Strand V, Birbara CA, Compagnone D, Fischkoff SA, Chartash EK. Adalimumab, a fully human anti tumor necrosis factor-alpha monoclonal antibody, and concomitant standard antirheumatic therapy for the treatment of rheumatoid arthritis: results of STAR (Safety Trial of Adalimumab in Rheumatoid Arthritis). J Rheumatol. 2003;30(12):2563-71.

Guo Y-R, Cao Q-D, Hong Z-S, Tan Y-Y, Chen S-D, Jin H-J, Tan K-S, Wang D-Y, Yan Y. The origin, transmission and clinical therapies on coronavirus disease 2019 (COVID-19) outbreakan update on the status. Mil Med Res. 2020;7(1):11. doi:10.1186/s40779-020-00240-0.

Grogono JC, Butler C, Izadi H, Moosavi SH. Inhaled furosemide for relief of air hunger versus sense of breathing effort: a randomized controlled trial. Respir Res. 2018;19(1):181-94. doi:10.1186/s12931-018-0886-9.

Gupta M, Lee HJ, Barden CJ, Weaver DF. The Blood-Brain Barrier (BBB) Score. J Med Chem. 2019;62(21):9824-36. doi:10.1021/acs.jmedchem.9b01220.

Gupta M, Bogdanowicz T, Reed MA, Barden CJ, Weaver DF. The Brain Exposure Efficiency (BEE) Score. ACS Chem Neurosci. 2020;11(2):205-24. doi:10.1021/acschemneuro.9b00650.

Peer) reviewing PDF | (2020:04:47843:1:2:NEW 5 Jun 2020) 
517 Hardman JG, Goodman LS, Gilman AG, editors. Goodman \& Gilman's The pharmacological

518 basis of therapeutics. 10 ${ }^{\text {th }}$ ed. New York, NY: McGraw-Hill Med. Publ; 2001.

519 Hosseinimehr SJ, Nobakht R, Ghasemi A, Pourfallah TA. Radioprotective effect of mefenamic 520 acid against radiation-induced genotoxicity in human lymphocytes. Radiat Oncol J.

521 2015;33(3):256-60. doi:10.3857/roj.2015.33.3.256.

522 Huang C, Wang Y, Li X, Ren L, Zhao J, Hu Y, Zhng L, Fan G, Xu J, Gu X, Cheng Z, Yu T, Xia 523 J, Wei Y, Wu W, Xie X, Yin W, Li H, Liu M, Xiao Y, Gao H, Guo L, Xie J, Wang G, Jiang R, 524 Gao Z, Jin Q, Wang J, Cao B. Clinical features of patients infected with 2019 novel coronavirus 525 in Wuhan, China. Lancet. 2020;395(10223):497-506. doi:10.1016/S0140-6736(20)30183-5.

526 Hung C-M, Peng C-K, Wu C-P, Huang K-L. Bumetanide attenuates acute lung injury by

527

528 suppressing macrophage activation. Biochem Pharmacol. 2018;156:60-7. doi:10.1016/j.bcp.2018.08.013.

529 Inokuchi R, Aoki A, Aoki Y, Yahagi N. Effectiveness of inhaled furosemide for acute asthma exacerbation: a meta-analysis. Crit Care. 2014;18(6):621-26. doi:10.1186/s13054-014-0621-y.

531 Lee W-S, Lee S-M, Kim M-K, Park S-G, Choi I-W, Choi I, Joo Y-D, Park S-J, Kang S-W, Seo

532

533 $\mathrm{S}-\mathrm{K}$. The tryptophan metabolite 3-hydroxyanthranilic acid suppresses $\mathrm{T}$ cell responses by

534 inhibiting dendritic cell activation. Int Immunopharmacol. 2013;17(3):721-6. doi:10.1016/j.intimp.2013.08.018.

535

536 Lia Q-J, Ye L-B, Timani KA, Zeng Y-C, She Y-L, Ye L, Wu Z-H. Activation of NF-kappaB by the Full-length Nucleocapsid Protein of the SARS Coronavirus. Acta Biochim Biophys Sin

537 (Shanghai). 2005;37(9):607-12. doi:10.111/j.1745-7270.2005.00082.x.

539

Lu Y-C, Yeh W-C, Ohashi PS. LPS/TLR4 signal transduction pathway. Cytokine. 2008;42(2):145-51. doi:10.1016/j.cyto.2008.01.006.

540 McGill University. Inhaled Nebulized Furosemide \& Physical Activity-Related Breathlessness.

541 https://ClinicalTrials.gov/show/NCT01851980 2016.

542 Mehta P, McAuley DF, Brown M, Sanchez E, Tattersall RS, Manson JJ. COVID-19: consider 543 cytokine storm syndromes and immunosuppression. Lancet. 2020;395(10229):1033-4.

544 doi:10.1016/S0140-6736(20)30628-0.

545 Molecular Operating Environment (MOE) 2019.0101; Chemical Computing Group ULC, 546 Montreal, QC, Canada, 2019.

547 National Cancer Institute Naples. Tocilizumab in COVID-19 Pneumonia (TOCIVID-19).

548 https://ClinicalTrials.gov/show/NCT04317092 2020.

549 O’Connor BJ, Chung KF, Chen-Worsdell YM, Fuller RW, Barnes PJ. Effect of inhaled 550 furosemide and bumetanide on adenosine 5'-monophosphate- and sodium metabisulfite-induced

551

552 bronchoconstriction in asthmatic subjects. Am Rev Respir Dis. 1991;143(6):1329-33. doi:10.1164/ajrccm/143.6.1329.

553 Oeckinghaus A, Hayden MS, Ghosh S. Crosstalk in NF-кB signaling pathways. Nat Immunol. 554 2011;12(8):695-708. doi:10.1038/ni.2065.

555 Oxford Brookes University. Specificity of Dyspnoea Relief With Inhaled Furosemide.

556 https://ClinicalTrials.gov/show/NCT02881866 2015. 
557 Panigrahi SK, Desiraju GR. Strong and weak hydrogen bonds in the protein-ligand interface.

558 Proteins. 2007;67(1):128-41. doi:10.1002/prot.21253.

559 Peking University First Hospital. Favipiravir Combined With Tocilizumab in the Treatment of

560 Corona Virus Disease 2019. https://ClinicalTrials.gov/show/NCT04310228 2020.

561 Plewczynski D, Lazniewski M, Augustyniak R, Ginalski K. Can we trust docking results?

562 Evaluation of seven commonly used programs on PDBbind database. J Comput Chem 2011;32

563 (4):742-55. doi:10.1002/jcc.21643

564 Prandota J. Furosemide: progress in understanding its diuretic, anti-inflammatory, and

565 bronchodilating mechanism of action, and use in the treatment of respiratory tract diseases. Am J

566 Ther. 2002;9(4):317-28. doi:10.1097/00045391-200207000-00009.

567 Qin C, Zhou L, Hu Z, Zhang S, Yang S, Tao Y, Xie C, Ma K, Shang K, Wang W, Tian D-S.

568 Dysregulation of immune response in patients with COVID-19 in Wuhan, China. Clin Infect Dis.

569 2020; ciaa248. doi:10.1093/cid/ciaa248.

570 Ramírez D, Caballero J. Is It Reliable to Take the Molecular Docking Top Scoring Position as

571 the Best Solution without Considering Available Structural Data?. Molecules. 2018;23(5):1038.

572 doi:10.3390/molecules23051038

573 Regeneron Pharmaceuticals, Sanofi. Evaluation of the Efficacy and Safety of Sarilumab in

574 Hospitalized Patients With COVID-19. https://ClinicalTrials.gov/show/NCT04315298 2020.

575 Sebba A. Tocilizumab: the first interleukin-6-receptor inhibitor. Am J Health Syst Pharm.

576 2008;65(15):1413-8. doi:10.2146/ajhp070449.

577 Tongji Hospital, Hubei Xinhua Hospital, Wuhan No.1 Hospital, Wuhan central hospital.

578 Tocilizumab vs CRRT in Management of Cytokine Release Syndrome (CRS) in COVID-19.

579 https://ClinicalTrials.gov/show/NCT04306705 2020.

580 University of Cologne. Trial on Treatment With Inhaled Furosemide of Preterm and Term

581 Neonates With Transient Tachypnoea. https://ClinicalTrials.gov/show/NCT01407848 2012.

582 University of North Carolina, Chapel Hill, Duke University, Eunice Kennedy Shriver National

583 Institute of Child Health and Human Development, The Emmes Company LLC. Safety of

584 Furosemide in Premature Infants at Risk of Bronchopulmonary Dysplasia (BPD).

585 https://ClinicalTrials.gov/show/NCT02527798 2015.

586 Waskiw-Ford M, Wu A, Mainra A, Marchand N, Alhuzaim A, Bourbeau J, Smith BM, Jensen D.

587 Effect of Inhaled Nebulized Furosemide (40 and $120 \mathrm{mg}$ ) on Breathlessness during Exercise in

588 the Presence of External Thoracic Restriction in Healthy Men. Front Physiol. 2018;9:86.

589 doi:10.3389/fphys.2018.00086.

590 Xu X, Han M, Li T, Sun W, Wang D, Fu B. Effective Treatment of Severe COVID-19 Patients

591 with Tocilizumab. Proc Natl Acad Sci USA. 2020;117(20):10970-5.

592 doi:10.1073/pnas.2005615117.

593 Yeo CT, O’Connor BJ, Chen-Worsdell M, Barnes PJ, Chung KF. Protective effect of loop

594 diuretics, piretanide and frusemide, against sodium metabisulphite-induced bronchoconstriction

595 in asthma. Eur Respir J. 1992;5(10):1184-8. 
596 Yuengsrigul A, Chin TW, Nussbaum E. Immunosuppressive and cytotoxic effects of furosemide 597 on human peripheral blood mononuclear cells. Ann of Allergy, Asthma Immunol.

598 1999;83(6):559-66. doi:10.1016/S1081-1206(10)62870-0. 


\section{Figure 1}

Screening approach with chemical structures of important compounds.

With the goal to find a small therapeutic with anti-inflammatory properties and endogenous to the human body, a library of 1,136 small molecules was screened by docking simulations and further investigated by in vitro experiments. This intensive screen yielded 3-

hydroxyanthranilic acid (3-HAA) as initial hit. Since 3-HAA is not approved for medical applications, 1,768 known drugs were screened computationally for a 3-HAA substructure. Besides mefenamic acid, which is not displayed in this figure, the diuretic furosemide was the strongest lead directly followed by bumetanide, azosemide and piretanide.
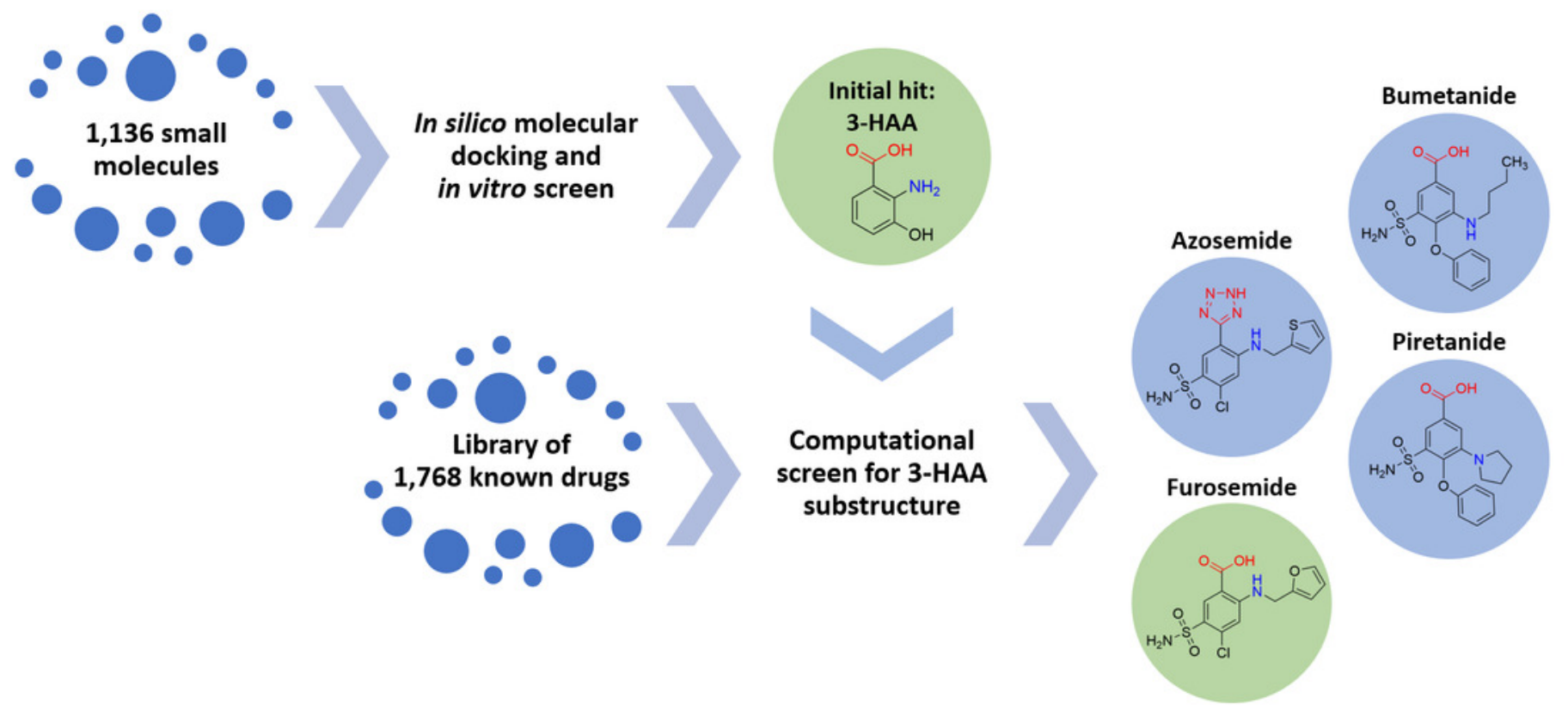


\section{Figure 2}

Furosemide decreases the production of NO and TNF- $\alpha$.

Production of (A) NO and (B) TNF- $\alpha$ from RAW264.7 cells upon LPS induction were determined by Griess assay and ELISA from the conditioned medium. Error bars show SEM, $\mathrm{n}=3 . *, p<$ 0.05. (C) iNOS expression level from RAW264.7 cells was assessed by Western blot analysis. Cells were treated with LPS+IFNy with DMSO or $25 \mu \mathrm{M}$ furosemide. After $24 \mathrm{~h}$ of incubation, cell lysate was harvested for Western blot analysis. GAPDH = Glyceraldehyde 3-phosphate dehydrogenase.

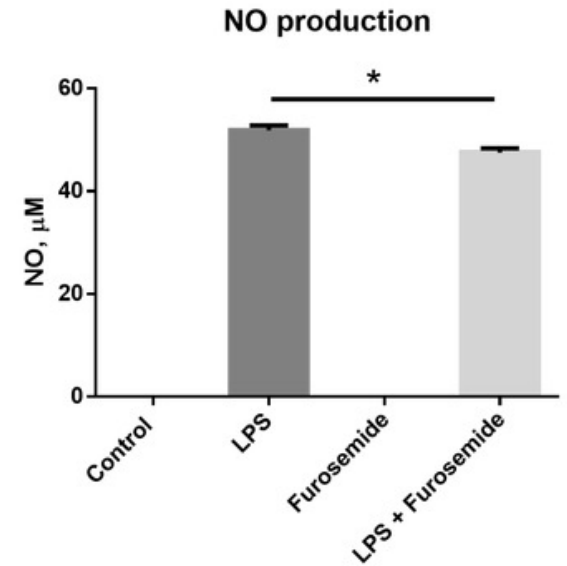

A

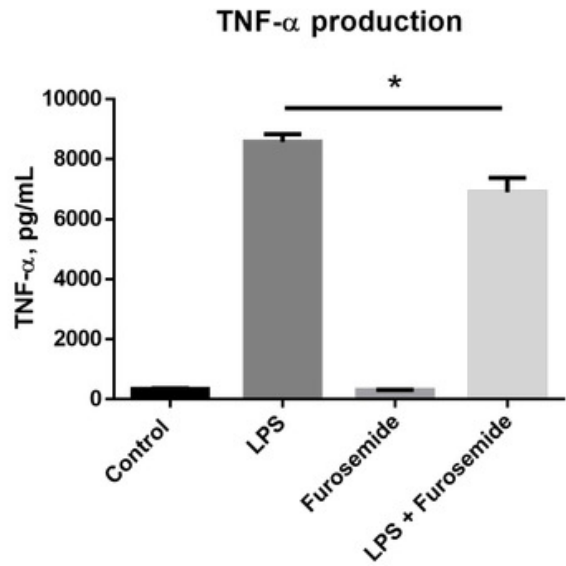

B

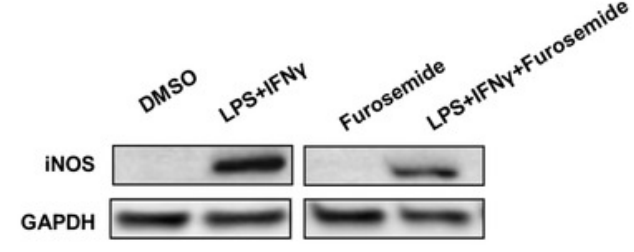

C 
Figure 3

Furosemide significantly decreases TLR4+ cell population.

RAW264.7 macrophage cells were stimulated with LPS and flow cytometry was used to determine TLR4+ cells population. (A), \% of Vis and (B), cell numbers for TLR4+. F = furosemide, FSC-A = front scatter.

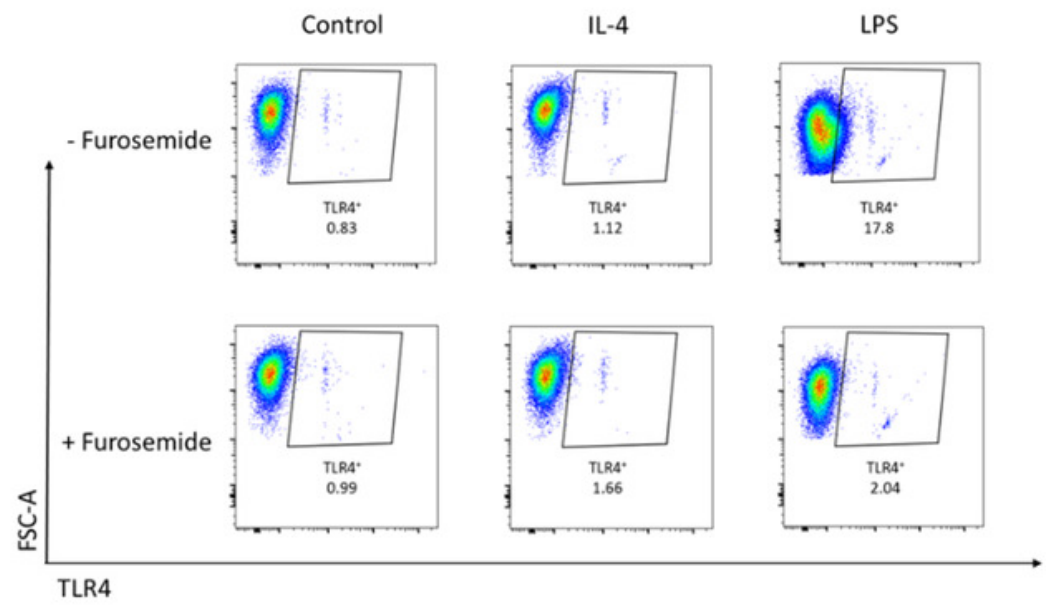

A

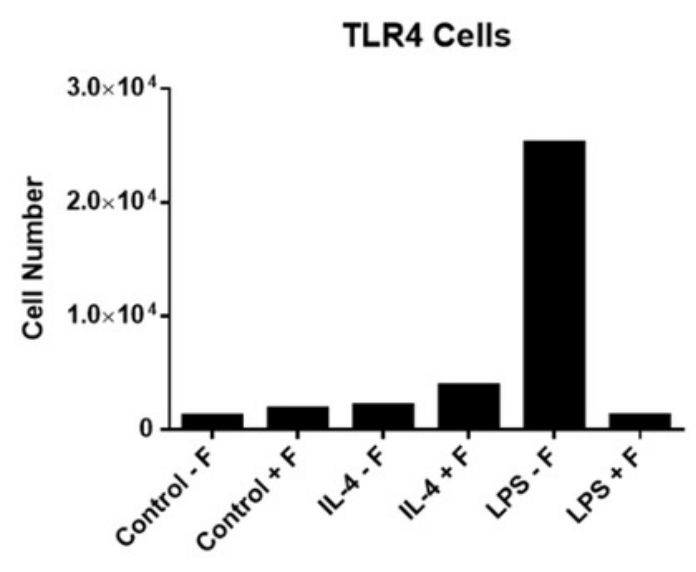

B 


\section{Figure 4}

Furosemide significantly inhibits pro-inflammatory responses.

Furosemide activity was initially tested on differentiated THP-1 macrophages. THP-1 monocytic cells were initially differentiated to THP-1 macrophages by PMA for $24 \mathrm{~h}$. Cells were then stimulated by LPS/IFNy in the presence of furosemide or DMSO control. Followed by $48 \mathrm{~h}$ incubation, the conditioned media and cell lysates were harvested for analysis. (A) Western blot showing the expression of pro-IL-1 $\beta$ upon treatment with DMSO and furosemide, respectively. Actin was used as a loading control. Productions of (B) NO, (C) IL-6 and (D) TNF$\alpha$ were measured by either Griess assay or ELISA. Error bars show SD, $\mathrm{n}=6 . * *, p<0.01$; ***, $p<0.001$.

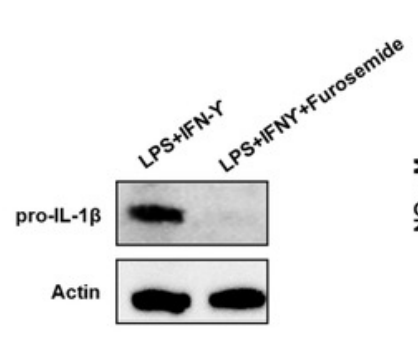

A

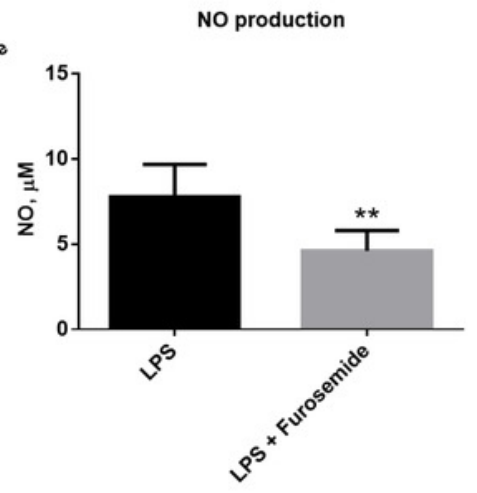

B
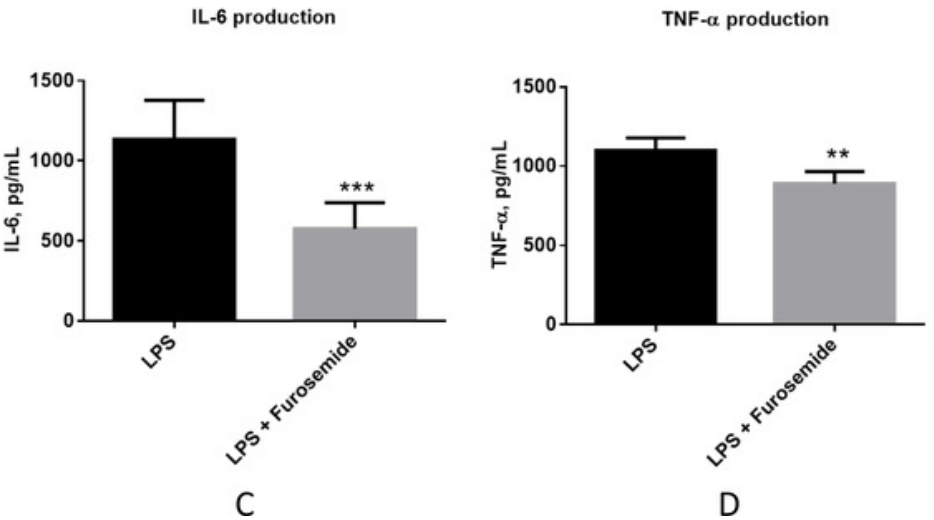


\section{Figure 5}

Furosemide induces the expression of anti-inflammatory phenotype markers on THP-1 macrophages.

The anti-inflammatory activity of furosemide was tested by THP-1 macrophages. THP-1 monocytic cells were initially differentiated to THP-1 macrophage by PMA for $24 \mathrm{~h}$. Cells were then stimulated by LPS/IFNy in the presence of furosemide or DMSO control. Followed by $48 \mathrm{~h}$ incubation, the conditioned media were harvested for (A) IL-4, (B) IL-IRA and (C) Arginase analysis. Error bars show SD, $\mathrm{n}=2 . * *, p<0.01$.
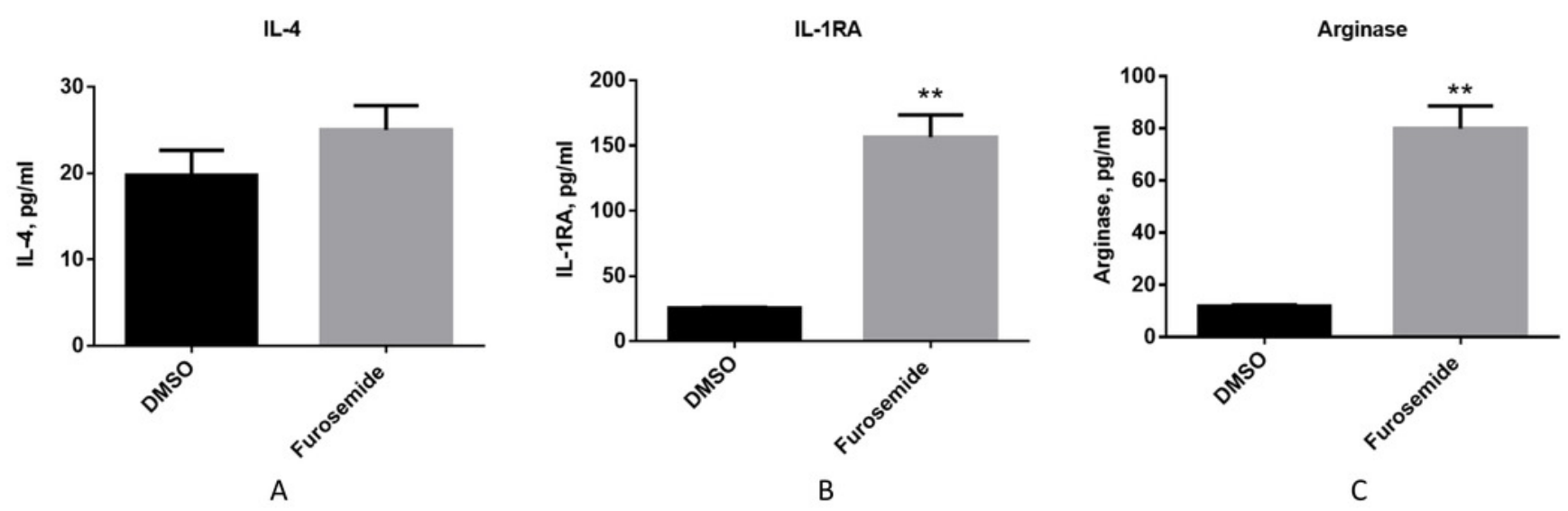
Figure 6

Docking simulations of co-crystallized ligand in IL-6.

(A) The overlay of docked conformer of tartaric acid against co-crystallized ligand conformation in IL-6 active site for validation of docking simulations. (B) Ligand interaction diagram of co-crystallized ligand 'tartaric acid' of IL-6 (PDB code: 1ALU) in re-docking simulation.

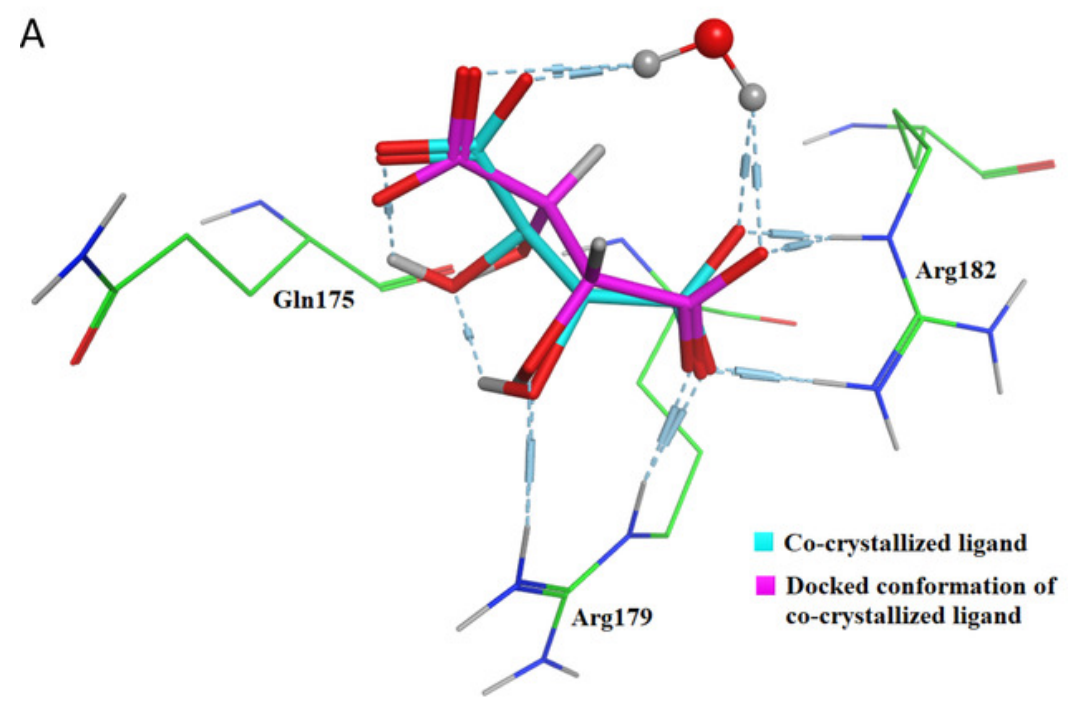

B

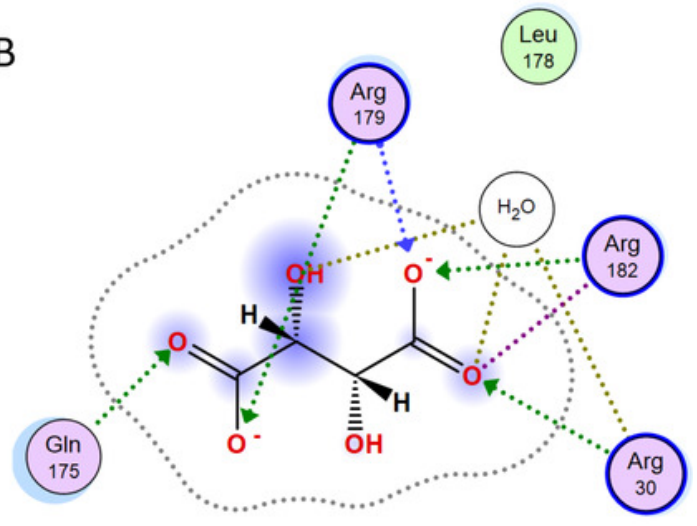


Figure 7

Docking simulations of co-crystallized ligand in TNF- $\alpha$.

(A) The overlay of docked conformer of TNF- $\alpha$ dimer inhibitor 307 against co-crystallized ligand conformation in TNF- $\alpha$ active site for validation of docking simulations. (B) Ligand interaction diagram of co-crystallized ligand of TNF- $\alpha$ dimer in a re-docking simulation.

A

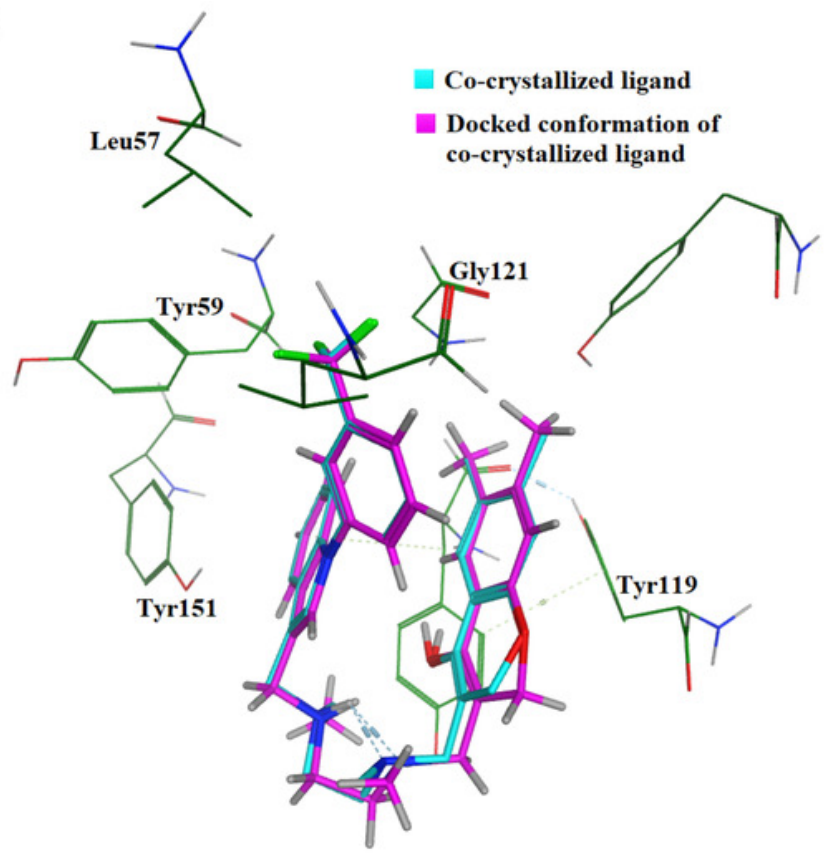

B

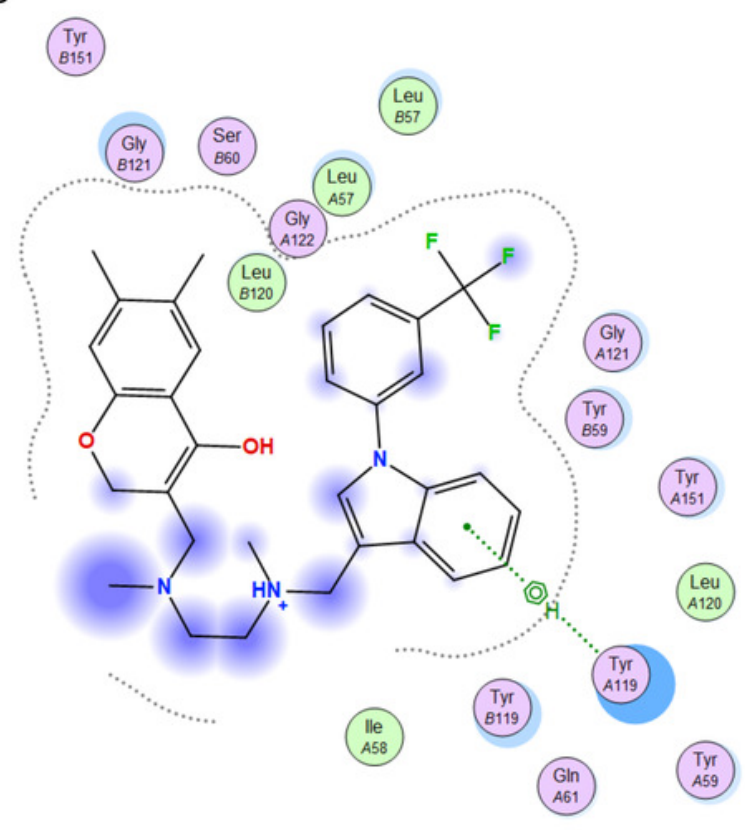


Figure 8

Interaction of 3-HAA with active site of TNF- $\alpha$ and IL-6.

(A) Binding of 3-HAA in the active site of tumor necrosis factor $\alpha$ (TNF- $\alpha$ ); (B) Ligand interaction diagram of 3-HAA in binding site of TNF- $\alpha$. (C) Binding of 3-HAA in the active site of interleukin-6 (IL-6); (C) Ligand interaction diagram of 3-HAA in binding site of IL-6.

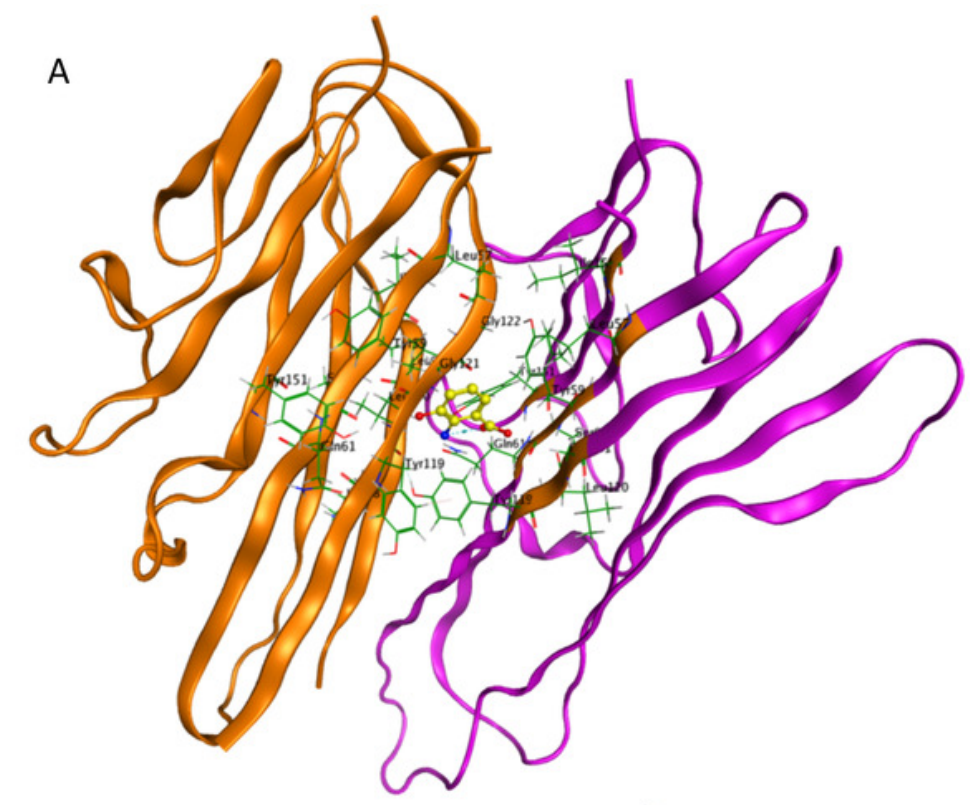

B

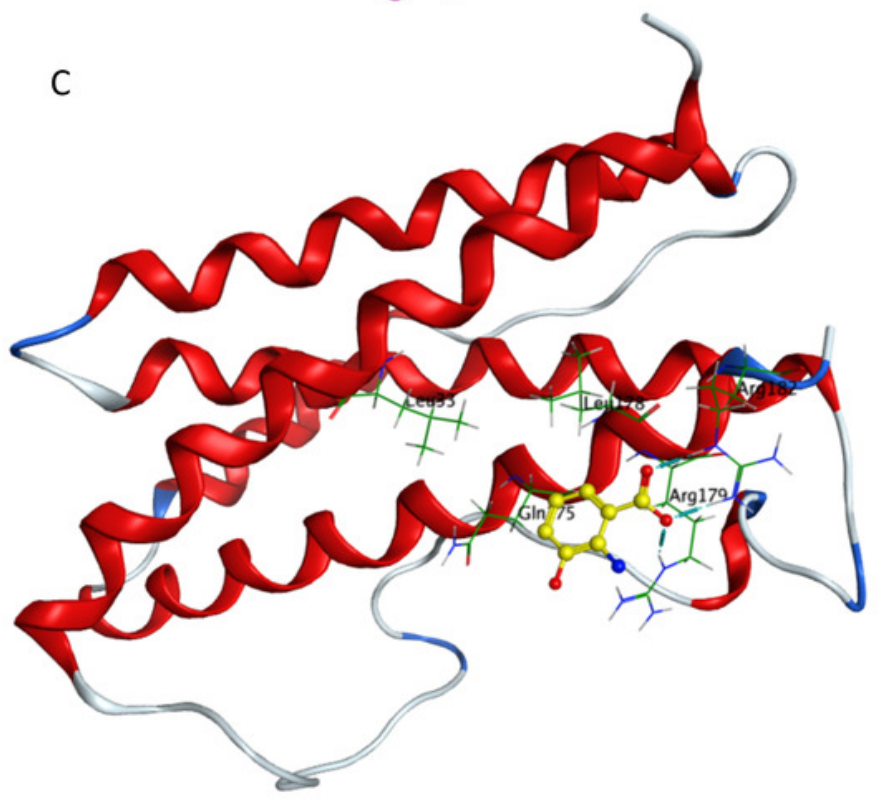

Tyr
A59
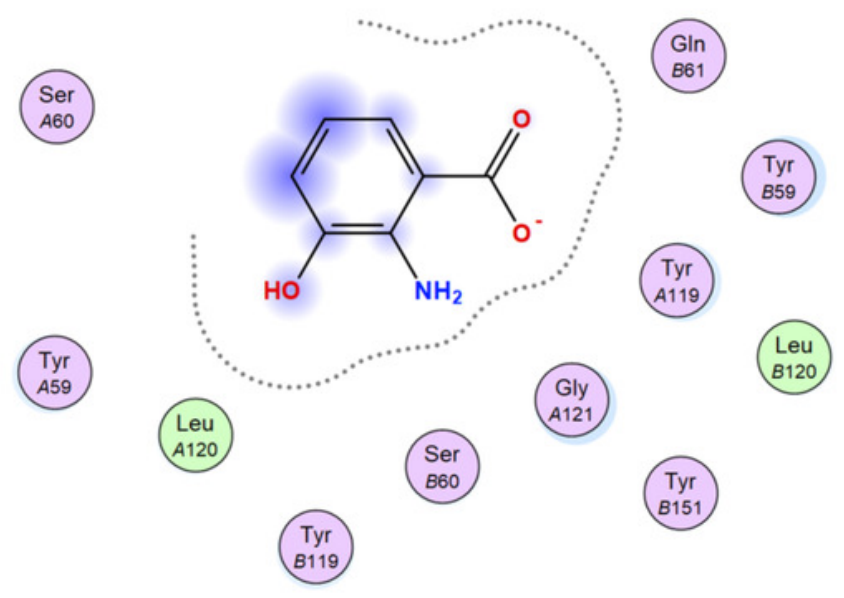

Leu

D

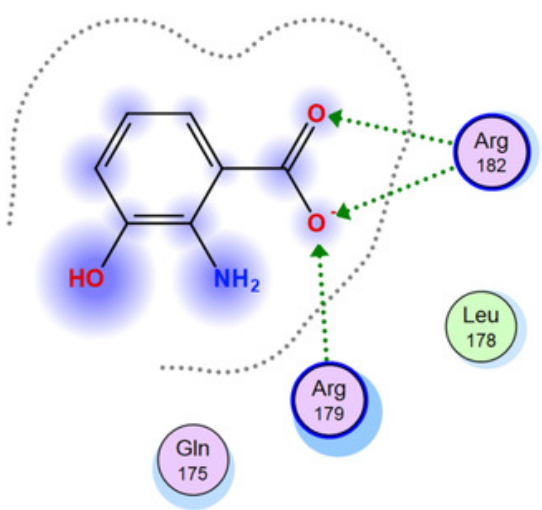


Figure 9

Interaction of furosemide with active site of TNF- $\alpha$ and IL- 6 .

(A) Binding of furosemide in the active site of tumor necrosis factor $\alpha$ (TNF- $\alpha$ ); (B) Ligand interaction diagram of furosemide in binding site of TNF- $\alpha$. (C) Binding of furosemide in the active site of interleukin-6 (IL-6); (D) Ligand interaction diagram of furosemide with binding site of IL-6. 

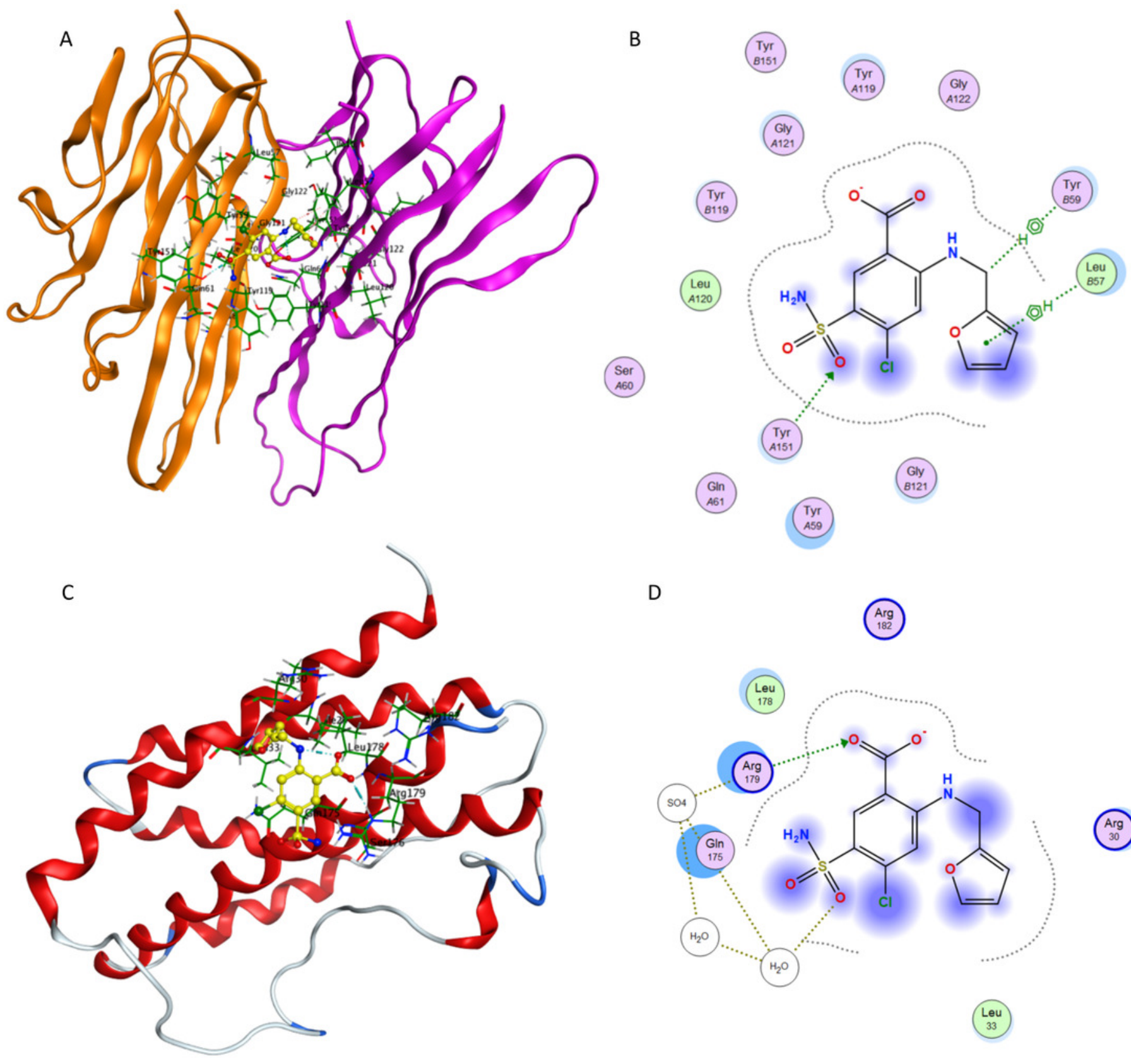

D

Arg

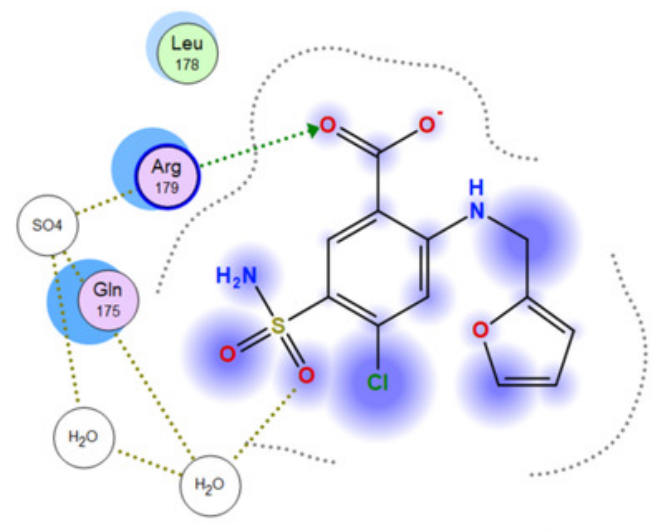

Arg

(Leu 


\section{Table $\mathbf{1}$ (on next page)}

Re-docking of the co-crystallized ligands tartaric acid and 307* in the active site of IL- 6 and TNF- $\alpha$.

The table includes RMSD data, interacting amino acid residues and binding energy values. 


\begin{tabular}{|c|c|c|c|c|c|c|}
\hline Protein & PDB ID & $\begin{array}{c}\text { Co-crystallized } \\
\text { Ligand }\end{array}$ & $\begin{array}{l}\text { Interacting } \\
\text { amino acid } \\
\text { residues in } \\
\text { the active } \\
\text { site }\end{array}$ & $\begin{array}{c}\text { Binding } \\
\text { pocket } \\
\text { residues } \\
\text { within } 4 \AA \\
\text { of radius }\end{array}$ & RMSD (Å) & $\begin{array}{c}\text { Binding } \\
\text { energy } \\
\text { (kcal/mol) }\end{array}$ \\
\hline IL-6 & $1 \mathrm{ALU}$ & Tartaric acid & $\begin{array}{l}\text { Gln175 } \\
\text { Arg179 } \\
\text { Arg182 }\end{array}$ & $\begin{array}{c}\text { Arg30 } \\
\text { Leu178 }\end{array}$ & 0.6114 & -4.7397 \\
\hline TNF- $\alpha$ & $2 \mathrm{AZ} 5$ & $\begin{array}{l}\text { Bound inhibitor- } \\
307^{*} \text { in TNF-a } \\
\text { dimer complex } \\
\text { form }\end{array}$ & Tyr119 & $\begin{array}{c}\text { Leu57 } \\
\text { Tyr59 } \\
\text { Ser60 } \\
\text { Leu120 } \\
\text { Gly121 } \\
\text { Gly122 } \\
\text { Tyr151 }\end{array}$ & 0.1677 & -7.5526 \\
\hline
\end{tabular}

1 *TNF- $\alpha$ dimer inhibitor 307 IUPAC name: 6,7-DIMETHYL-3-[(METHYL $\{2-[M E T H Y L(\{1-[3-$

2 (TRIFLUOROMETHYL)PHENYL]-1H-INDOL-3-YL\}METHYL)AMINO]ETHYL \} AMINO) METHYL]-4H-

3 CHROMEN-4-ONE.

4 